\title{
Autoregulation of fixK (2) gene expression in Bradyrhizobium japonicum
}

\section{Journal Article}

\section{Author(s):}

Reutimann, Luzia; Mesa, Socorro; Hennecke, Hauke

Publication date:

2010-07

Permanent link:

https://doi.org/10.3929/ethz-b-000020563

\section{Rights / license:}

In Copyright - Non-Commercial Use Permitted

\section{Originally published in:}

Molecular genetics and genomics 284(1), https://doi.org/10.1007/s00438-010-0547-2 


\title{
Autoregulation of fix $K_{2}$ gene expression in Bradyrhizobium japonicum
}

\author{
Luzia Reutimann · Socorro Mesa · Hauke Hennecke
}

Received: 20 April 2010/Accepted: 19 May 2010/Published online: 4 June 2010

(C) Springer-Verlag 2010

\begin{abstract}
Several essential Bradyrhizobium japonicum genes for a symbiotic, nitrogen-fixing root-nodule symbiosis are positively controlled under micro-oxic conditions by the FixLJ-FixK ${ }_{2}$ regulatory cascade. Negative control is exerted by reactive oxygen species at the level of the FixK 2 protein. Furthermore, we noticed that fix $K_{2}$ gene expression is increased in a fix $K_{2}$ mutant, suggesting that Fix $\mathrm{K}_{2}$ in the wild type has a negative effect, directly or indirectly, on its own expression. To possibly understand this effect, the transcription pattern of the fixLJ-bll2758-fix $K_{2}$ gene region was examined more closely. While $f i x K_{2}$ gene transcription is activated by FixJ, the bll2758 gene is transcribed from its own promoter in a FixK ${ }_{2}$-dependent manner, and there is no read-through transcription from bll2758 into fix $K_{2}$. The bll2758-encoded protein is predicted to be a stand-alone receiver domain of a response regulator, making it a prime candidate for exerting an inhibitory role on the expression of fix $K_{2}$. Transcriptome profiling of a bll2758 knock-out mutant revealed, however, that neither fix $K_{2}$ itself nor any of the known FixJ- and FixK $_{2}$-dependent target genes is significantly affected in their expression. This precludes a role of the bll2758 product as a so-called FixT-like protein in the inhibition of FixLJ function, as was proposed for Sinorhizobium meliloti and Caulobacter crescentus. Instead, we rationalize that other transcription factors, whose genes are activated by FixK $\mathrm{K}_{2}$, might be involved in the negative autoregulation of fix $K_{2}$ gene expression.
\end{abstract}

Communicated by G. Klug.

L. Reutimann $\cdot$ S. Mesa $\cdot$ H. Hennecke $(\bowtie)$

ETH, Institute of Microbiology, Wolfgang-Pauli-Strasse 10, 8093 Zürich, Switzerland

e-mail: hennecke@micro.biol.ethz.ch
Keywords Bradyrhizobium japonicum · Gene regulation · Microarrays $\cdot$ Promoter $\cdot$ Transcription
Abbreviations
cDNA Complementary DNA
FixJ-P Phosphorylated FixJ
ORF Open reading frame
PCR Polymerase chain reaction

\section{Introduction}

Strong induction of bacterial gene expression is often achieved either when target genes are subject to dual positive control, involving two synergistically acting, disparate activators, or when two or more transcription activators work sequentially in a cascade in which the first induces expression of the gene for the second, thus boosting the level of the ultimate activator of target genes. These arrangements also allow the integration of additional regulatory cues at different levels. The FixLJ-FixK cascade, addressed in this work, which exists in many symbiotic, nitrogen-fixing rhizobia, is an example of a sequentially acting pathway (for reviews, see Fischer 1994; Batut and Boistard 1994; Dixon and Kahn 2004). FixL, a sensorhistidine autokinase, responds to a decreased oxygen tension and subsequently phosphorylates the response regulator FixJ. FixJ-phosphate (FixJ-P) then activates the gene for FixK, a member of the CRP/FNR protein family. FixK, in turn, activates genes and operons related to micro-oxic energy metabolism, e.g., the fixNOQP and fixGHIS operons for the synthesis and function of the high-affinity $c b b_{3}$-type oxidase. 
In contrast to the gene activation steps in the FixLJ-FixK cascade, less information is available about how the genes are down-regulated. Four negatively interfering modes of control have been described for either Sinorhizobium meliloti or Bradyrhizobium japonicum: (1) decrease of the FixJ-P level by oxygenation of FixL (Lois et al. 1993; Sousa et al. 2007); (2) negative autoregulation of fixK gene expression (Foussard et al. 1997; Nellen-Anthamatten et al. 1998; Garnerone et al. 1999); (3) inhibition of fixK gene expression by a precursor of purine biosynthesis (Soberón et al. 2001) and; (4) posttranslational control of FixK 2 activity by reactive oxygen species (Mesa et al. 2009). The mechanism of fixK autoregulation in S. meliloti is indirect (Fig. 1a). The FixK protein activates the $f i x T_{1}$ gene, encoding a stand-alone receiver-domain protein that was shown to inhibit FixL autophosphorylation (Foussard et al. 1997; Garnerone et al. 1999). The anti-kinase activity of FixT 1 seems to be assisted by an asparagine synthetase-like protein (AsnO), but the physiological meaning of this kind of control remains to be elucidated (Bergès et al. 2001). Many elements of the S. meliloti fixK autoregulation mechanism were found to operate in a similar way in the non-symbiotic, nitrogen non-fixing Caulobacter crescentus (Crosson et al. 2005; Paul et al. 2008; Jenal and Galperin 2009), although the precise site of inhibition by FixT is not known (Fig. 1b). A fixT-like gene (bll2758) was also identified previously in B. japonicum (Nellen-Anthamatten et al. 1998; Kaneko et al. 2002), where it is located between the fix $L J$ and fix $K_{2}$ genes (Fig. 1c). The aim of this work was to unravel the transcriptional organization of the bll2758-fix $K_{2}$ region and to find out whether the bll2758 product functions like the FixT protein. The result was that $B$. japonicum differs in this respect from $S$. meliloti and $C$. crescentus. Although we could demonstrate Fix $_{2}$-dependent transcription activation of the bll2758 gene, the product of the latter does not appear to interfere with the expression of either fix $K_{2}$ or known FixK $\mathrm{K}_{2}$-dependent genes. An alternative scenario for the negative autoregulation of $f i x K_{2}$ is discussed.

\section{Materials and methods}

Media and growth conditions

Bradyrhizobium japonicum strains were grown either oxically with vigorous shaking or micro-oxically $\left(0.5 \% \mathrm{O}_{2}\right.$ and $99.5 \% \mathrm{~N}_{2}$ in the gas phase) in a modified peptone-salts-yeast extract medium (Mesa et al. 2008). The growth temperature in all experiments was $30^{\circ} \mathrm{C}$. Where appropriate, cultures contained the following concentrations of antibiotics (in $\mu \mathrm{g}$ per $\mathrm{mL}$ ): spectinomycin, 100; kanamycin, 100. Escherichia coli strains harboring recombinant plasmids

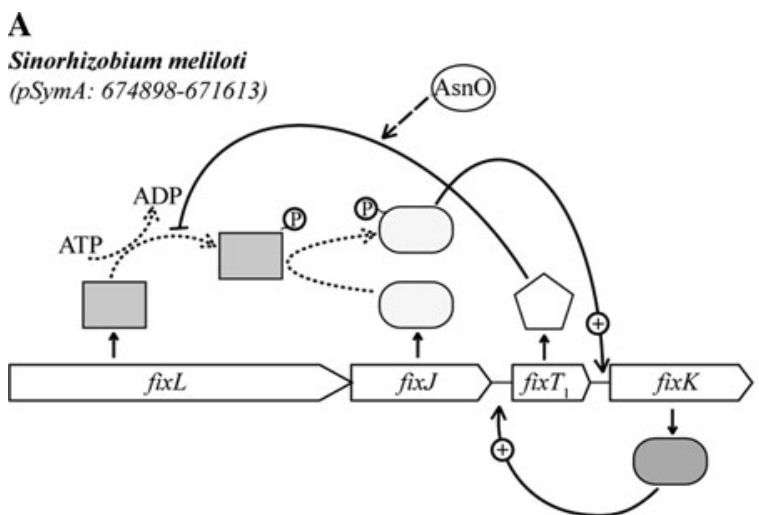

B

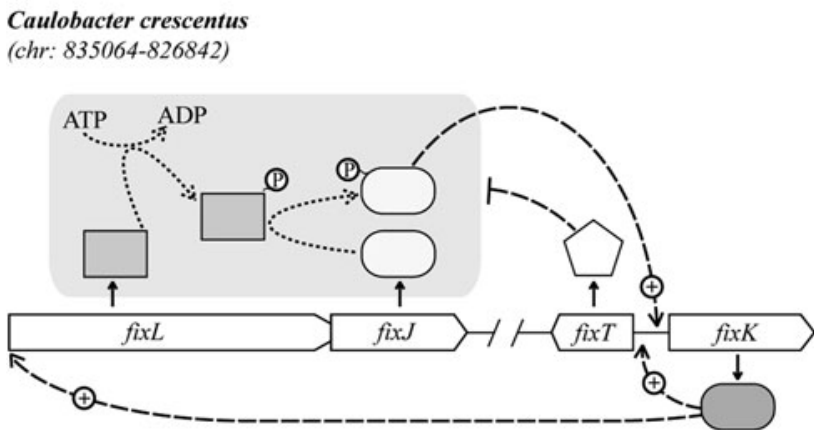

C

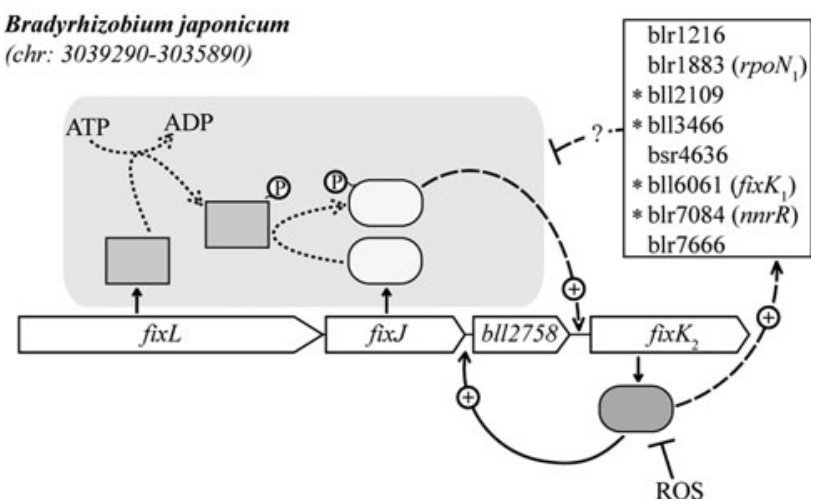

Fig. 1 Comparison of FixLJ-FixK regulatory circuits in S. meliloti (a), C. crescentus (b), and B. japonicum (c). The scheme for B. japonicum reflects work elaborated not only previously but also in this work. See text for a description of the three models. Genes are drawn to scale. Their genome coordinates are shown below species names. Specific geometric shapes are used for homologous gene products. The large gray-shaded rectangle in $\mathbf{b}$ and $\mathbf{c}$ means that, in contrast to a, the precise site of negative autoregulation is not known. The stand-alone receiver domain encoded by fixT/bll2758 appears to play a negative regulatory role only in a and b. Instead, other transcription factors compiled in a box may play this role in c. Of these, CRP/FNR family members are marked with an asterisk. Solid lines: biochemically proven direct interactions. Dashed lines: direct or indirect interactions. Dotted lines: phosphoryl group transfer. Lines ending with a circled + and/or arrowheads symbolize positive control. Lines ending with a $T$ bar symbolize negative control. ROS, reactive oxygen species 
were grown in Luria-Bertani medium at $37^{\circ} \mathrm{C}$ (Miller 1972). Antibiotics were used as follows (in $\mu \mathrm{g}$ per $\mathrm{mL}$ ): ampicillin, 200; kanamycin, 30.

\section{Bradyrhizobium japonicum strains}

Bradyrhizobium japonicum strains used in this work were $110 s p c 4$ (wild type, Regensburger and Hennecke 1983); 7360 (fixJ::aphII, Anthamatten and Hennecke 1991); 9043 ( $\Delta$ fix $K_{2}$, Nellen-Anthamatten et al. 1998); 7454 (fix $K_{1}:: a p h I I$, Anthamatten et al. 1992); 7414 (bll2758::aphII, Anthamatten and Hennecke 1991); 8678 ( $\Delta n n r R$, Mesa et al. 2003); and N50-97 (rpoN $N_{1}:: a p h I I+r p o N_{2}:: \Omega$, Kullik et al. 1991). Insertion of a translational fix $K_{2}^{\prime}-^{\prime}$ lac $Z$ fusion into the chromosomes of the wild type, the $\Delta$ fix $K_{2}$ mutant, and the $\Delta n n r R$ mutant gave rise to strains 9054 (Nellen-Anthamatten et al. 1998), 9054K2 (Nellen-Anthamatten et al. 1998), and 9054R (this work), respectively. $\beta$-Galactosidase activity in these strains was determined as described previously (Fischer et al. 1993).

\section{Reverse transcription PCR}

The technique was used to determine if the two adjacent genes bll2758 and fix $K_{2}$ are transcribed as an operon. RNA template from micro-oxically grown $B$. japonicum wildtype cells was reverse-transcribed to cDNA with the same method as used in microarray sample preparation (Hauser et al. 2007). Subsequently, the cDNA was used as template for a hot-start PCR with Pfu DNA polymerase. The conditions were $30 \mathrm{~s}$ at $94^{\circ} \mathrm{C}$ for melting; $60 \mathrm{~s}$ at $60^{\circ} \mathrm{C}$ for annealing; and $90 \mathrm{~s}$ at $68^{\circ} \mathrm{C}$ for elongation. 35 cycles were run. The following primers were employed in different pairwise combinations: LR15, 5'-TCTCCTCGTCCTTGC GGTAG; LR16， 5'-TAGCCGGTGATCAGGATCACG; LR17, 5'-CAACGGAGATGGCGCAGATG; LR19, 5'-GC TGGAAACCGACGGCTTTG. Their positions and orientations are depicted in Fig. 2.

In vitro transcription

Assays for multiple-round in vitro transcription were carried out in a volume of $20 \mu \mathrm{l}$ under standard conditions as described previously (Beck et al. 1997; Mesa et al. 2005). Plasmids used as transcription templates were based on pRJ9519 which contains the B. japonicum rrn terminator (Beck et al. 1997). A 210-bp EcoRI fragment with the bll2758 promoter region was cloned into pRJ9519, resulting in plasmid pRJ0208. The fixN promoter-containing plasmid pRJ8816 (Mesa et al. 2005) was applied as a positive-control template. Purified FixK $_{2}$ protein (Mesa et al. 2005) was used in amounts of 1.25 or $2.5 \mu \mathrm{M}$. RNA size markers were prepared as described (Mesa et al. 2005).
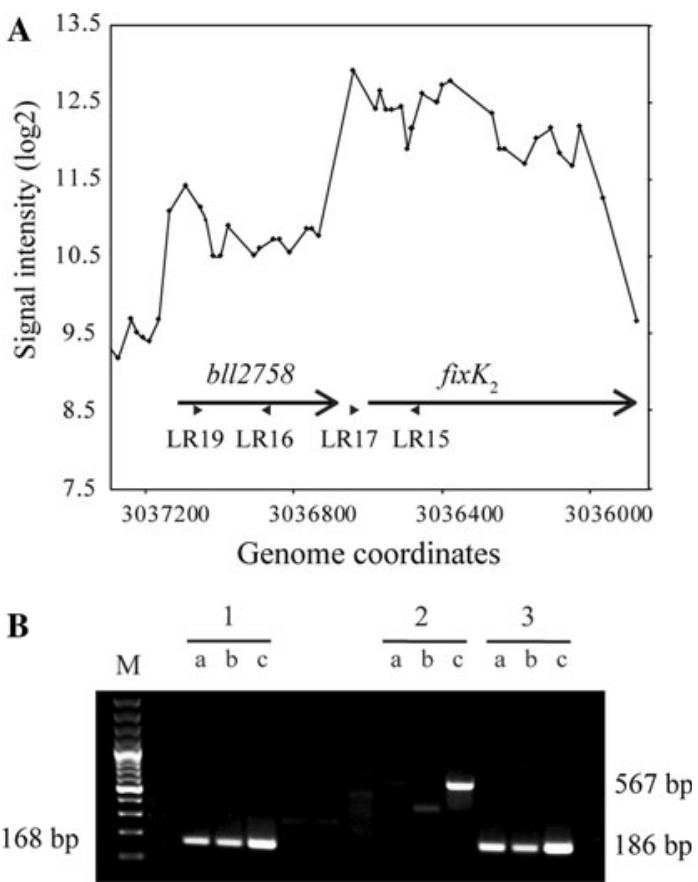

Fig. 2 Transcriptional organization of the bll2758-fix $K_{2}$ locus in B. japonicum. a Signal intensities derived from different oligonucleotide probes of the bll2758-fix $K_{2}$ region on the Gene Chip microarray. Data were obtained with RNA from micro-oxically grown wild-type cells. b Reverse transcription PCR. LR15, LR17, LR16, LR19 plus the associated small, filled arrowheads denote the binding sites and orientations of primers. cDNA was obtained from RNA of microoxically grown wild type. Lanes labeled with $a, 50 \mathrm{ng}$ cDNA; lanes labeled with $b, 5 \mathrm{ng}$ cDNA; lanes labeled with $c, 50 \mathrm{ng}$ genomic DNA (control). Primer combinations are indicated above the gel: 1 LR15 + LR17, 2 LR15 + LR19, 3 LR16 + LR19. Lengths (in bp) are indicated next to the respective band. $M$ markers

A phosphoimager (Imager FX Pro; Bio-Rad Laboratories, Hercules, CA, USA) was used to detect the transcripts. For visualization, Quantity One Software, Version 4.6.7 (BioRad Laboratories), was applied.

Primer-extension experiments

The transcription start site of bll2758 was determined with methods reported previously (Beck et al. 1997; Mesa et al. 2005). Primer 9519-1 (5'-ATGGATGAACTCCAAGACG GTATC) was used for primer extension with the transcript synthesized in vitro from plasmid pRJ0208. To determine the transcription start site of bll2758 in vivo, RNA template was extracted from micro-oxically grown $B$. japonicum wild-type cells, and primer LR21 (5'-GATCCCAGG ACTGCGGCGTCATCATC) was used for extension.

Microarrays

Genome-wide transcription levels were determined as detailed previously, using a custom-designed $B$. japonicum 
Affymetrix Gene Chip (Hauser et al. 2007; Mesa et al. 2008, 2009). In this work, the transcription profile of strain 7414 (bll2758::aphII) was analyzed. The complete dataset has been deposited in the Gene Expression Omnibus database (GEO; http://www.ncbi.nlm.nih.gov/geo) under GEO Series accession number GSE21400. Tiling analysis was done as described by Hauser et al. (2007) using the Affymetrix Tiling Analysis Software (Affymetrix, Santa Clara, CA, USA).

\section{Bioinformatics}

Potential FixK $_{2}$ binding sites in DNA sequences were identified by searching for TTG- $\mathrm{N}_{8}$-CA motifs and by searching for sequences containing the TTG stretch and at least three additional nucleotides of the consensus TTGAT$\mathrm{N}_{4}$-ATCAA (Mesa et al. 2005). The program T-COFFEE (http://www.ebi.ac.uk/Tools/t-coffee/index.html) was used for the alignment of the predicted 118-amino-acid Bl12758 protein with other stand-alone receiver domains. The result was then visualized with GeneDoc 2.7 (http://www.psc. edu/biomed/genedoc).

\section{Results}

The bll2758 and fix $K_{2}$ genes are not cotranscribed

The organization of the B. japonicum fixL-fixJ-bll2758$f_{i x} K_{2}$ gene region is shown in Fig. 1c. The Kazusa nomenclature is used for bll2758 (http://genome.kazusa. or.jp/rhizobase) because, for reasons given below, the previous designation ORF138 (Anthamatten and Hennecke 1991) was found to be inappropriate. A FixJ-P-activated promoter is located in front of fix $K_{2}$ (Nellen-Anthamatten et al. 1998). It was noticed previously that expression of a chromosomally integrated $f i x K_{2}{ }^{\prime}{ }^{\prime}$ lac $Z$ fusion led to a more than tenfold higher $\beta$-galactosidase activity in a fix $K_{2}$ knock-out mutant as compared with the wild-type background, when cells of both strains had been grown under micro-oxic conditions (Nellen-Anthamatten et al. 1998). This observation was confirmed after inspection of data recently obtained by microarray-assisted transcriptome analyses: again, transcription from the fix $K_{2}$ promoter was found to be up-regulated in a fix $K_{2}^{-}$background (Mesa et al. 2008). This suggested that FixK $K_{2}$ regulates its own structural gene, directly or indirectly, in a negative way. Therefore, as a first approximation, the DNA upstream and around the beginning of fix $K_{2}$ was examined for the presence of FixK $\mathrm{K}_{2}$ binding sites, using the canonical FixK ${ }_{2}$ box $\left(5^{\prime}\right.$-TTGAT- $\mathrm{N}_{4}$-ATCAA- $\left.3^{\prime}\right)$ as the query sequence (Mesa et al. 2005, 2008), although we did not know whether a repressor-binding site would look similar to the bona fide activator binding site. One sequence (5'-TTGAG- ${ }_{4}$ GTCAA- $3^{\prime}$ ) that complied best with the consensus was identified at the beginning of bll2758. We reasoned that FixK $_{2}$ bound to that site could exert a negative effect on fix $K_{2}$ expression only if there was a read-through transcription from bll2758 into fix $K_{2}$. The results of experiments shown in Fig. 2 clearly demonstrate that this is not the case. The tiling-like architecture of the Gene Chip used (Hauser et al. 2007) helped reveal a non-uniform transcription intensity throughout this region, with a marked increase of transcription at the start of $f i x K_{2}$ (Fig. 2a). More importantly, using reverse transcription PCR, we did not detect RNA spanning the bll2758 and fix $K_{2}$ genes (Fig. 2b, lanes $2 a$ and $2 b$, with the primer pair LR15/LR19), whereas individual transcripts from within fix $K_{2}$ (lanes $1 \mathrm{a}$ and $1 \mathrm{~b}$ ) and bll2758 (lanes 3a and 3b) were detected. Hence, bll2758 and fix $K_{2}$ are transcribed as separate genes, not as an operon.

The $b l l 2758$ gene is a new direct target for activation by FixK $_{2}$

Although the putative Fix $\mathrm{K}_{2}$ box described above was very near the translational start of the annotated bll2758 ORF, we suspected that it might rather be a promoter element than a repressor binding site. To clarify the issue, the possible activation of the bll2758 gene by FixK $_{2}$ was first tested in a cell-free transcription assay (Mesa et al. 2005) with $B$. japonicum RNA polymerase holoenzyme and purified $\mathrm{FixK}_{2}$, and using the bll2758 5'-region as DNA template. The result is shown in Fig. 3. A bll2758-specific transcript was synthesized only when the FixK $_{2}$ protein was present in the assay (lanes 2 and 3). Accordingly, this gene is a newly identified direct target for transcription activation by FixK $_{2}$, thus expanding the list of 11 approved FixK $_{2}$ targets (Mesa et al. 2008) by one additional member. Next, the transcription start site of bll2758 was determined by primer extension (Fig. 4). Both, the in vitro synthesized RNA and the RNA extracted from micro-oxically grown cells gave rise to an extension product whose end mapped to a $\mathrm{G}$ (position +1 ; Fig. 4a, b) just two nucleotides before the annotated start codon of the bll2758 ORF (Fig. 4c). This is too close to accommodate a ribosome binding site. Most likely, therefore, the true translation start is 20 codons further downstream at a GTG triplet (Fig. 4c), and the ORF contains 118 instead of 138 codons. ORF138, the name coined previously for bll2758 (Anthamatten and Hennecke 1991; Nellen-Anthamatten et al. 1998), was henceforth abandoned. The -10 and -35 regions upstream of the transcription start site possess reasonably well conserved promoter sequences. Of particular interest is the partial overlap of the -35 region with the aforementioned FixK $_{2}$ box, as depicted in Fig. 4b. This arrangement is 


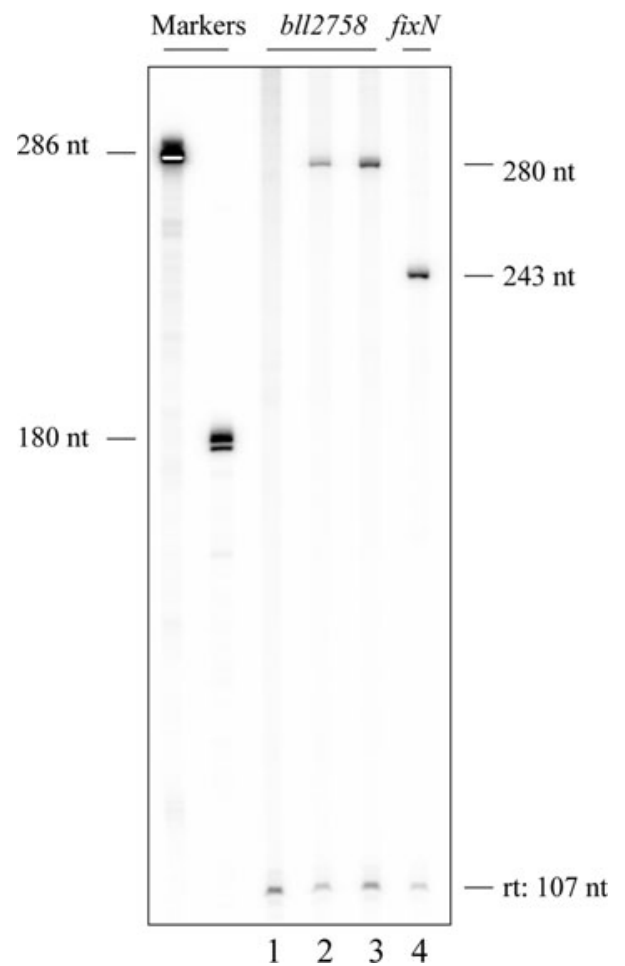

Fig. 3 In vitro transcription from the bll2758 promoter. Transcripts from template plasmid pRJ0208, which contains the bll2758 promoter region, were generated by multiple-round in vitro transcription with purified B. japonicum RNA polymerase holoenzyme and purified FixK $_{2}$ protein. Plasmid pRJ8816, which contains the promoter of the FixK $\mathrm{K}_{2}$-dependent fixN gene was used as control. Markers were generated as described previously (Mesa et al. 2005). FixK $\mathrm{K}_{2}$ protein concentrations were as follows: lane 1, no protein added; lanes 2 and 4, $1.25 \mu \mathrm{M}$; lane 3, $2.5 \mu \mathrm{M}$. The positions of the FixK $\mathrm{K}_{2}$-dependent transcripts, the FixK $\mathrm{K}_{2}$-independent vector-encoded reference transcript (rt), and the two RNA size markers are labeled; $n t$ nucleotides

characteristic for all promoters of Fix $\mathrm{K}_{2}$-activated genes (Mesa et al. 2008). Taken together, FixK $\mathrm{K}_{2}$ positively controls bll2758 (Fig. 1c), in a similar way as fixT is regulated by FixK in S. meliloti and C. crescentus (Fig. 1a, b).

Unlike FixT, the bll2758 product is not a fix $K_{2}$-specific negative regulator

With the newly proposed translational start at the GTG codon (Fig. 4c), the bll2758 gene codes for a predicted 118-amino acid protein that shares a low, but significant sequence similarity with previously described FixT-like single-domain response regulators (Jenal and Galperin 2009) of different proteobacteria (Fig. 5). Among several functionally conserved amino acids is a strictly conserved aspartic acid residue as the site of phosphorylation (Fig. 5). Being devoid of DNA-binding domains, such proteins do not directly affect transcription but modulate gene expression indirectly by interacting with other regulators. S. meliloti Fix $_{1}$, for example, inhibits FixL autokinase activity (Foussard et al. 1997; Garnerone et al. 1999). To unravel any effect on the expression of fix $K_{2}$ and FixK $_{2}$ dependent genes, the bll2758 mutant (strain 7414) grown micro-oxically was investigated with the sensitive microarray-assisted transcriptome profiling. The wild type and a fix $K_{2}$ mutant (strain 9043) were compared as controls. The rationale was that if the B112758 protein were a FixT-like negative effector in the wild type, the bll2758 mutant would display a de-repression of Fix $\mathrm{K}_{2}$ targets. This was clearly not the case (Table 1). Expression of none of the approved Fix $\mathrm{K}_{2}$-activated genes resulted in positive foldchange values in strain 7414 as compared with the wild type (Table 1). Likewise, expression of the fix $K_{2}$ gene itself and of the few other FixJ-P-dependent genes identified previously (Mesa et al. 2008) was unaltered (data not shown). This speaks against a negative regulatory role of B112758 in B. japonicum, as opposed to the role of FixTlike proteins in S. meliloti and C. crescentus (Fig. 1).

Negative fix $K_{2}$ autoregulation is possibly mediated by other transcription factors

Lack of evidence for a direct repression of the fix $K_{2}$ gene by the $\mathrm{FixK}_{2}$ protein led us to assume that autoregulation is indirect. As B112758 was excluded here, which other protein might be involved in negative control? A close examination of the comprehensive $\mathrm{FixK}_{2}$ regulon elaborated by Mesa et al. (2008) uncovered a total of eight genes that code for transcription factors (see box in Fig. 1c). At least one of them might be a candidate to work either as a repressor or as an activator of a repressor gene. The $\sigma^{54}$ gene $r p o N_{1}\left(\right.$ blr1883) as well as the fix $K_{1}$ and $n n r R$ genes (bll6061and blr7084, respectively) were known to us from previous work (Kullik et al. 1991; Anthamatten et al. 1992; Nellen-Anthamatten et al. 1998; Mesa et al. 2003). Since the $\sigma^{54}$ and Fix $_{1}$ regulons of micro-oxically grown cells were available from genome-wide transcriptomics studies (Hauser et al. 2007; Mesa et al. 2008), it was possible to examine the $f i x K_{2}$ gene and all of the 11 direct FixK $K_{2}$ targets for any de-repression in $\mathrm{rpoN}^{-}$and $f i x K_{1}^{-}$backgrounds, similarly as this was done with the bll2758 ${ }^{-}$strain (cf. Table 1). Neither fix $K_{2}$ itself nor the 11 targets showed altered expression in the $r p o N^{-}$strain N50-97 (data not shown), which argues against them being repressed by a $\sigma^{54}$-dependent repressor in the wild-type. Likewise, none of the genes in question was de-repressed in the fix $K_{1}^{-}$ strain 7454 (data not shown), arguing against FixK $_{1}$ as a potential repressor mediating indirect autoregulation of fix $K_{2}$ in the wild type. To test the NnrR protein as a potential repressor for fix $K_{2}$, we measured the expression of a chromosomally inserted fix $K_{2}^{\prime}{ }^{\prime}$ lacZ fusion in an $n n r R^{-}$ mutant and, for control, in the wild type and a fix $K_{2}^{-}$mutant (Fig. 6). The $n n r R$ mutant showed no difference in $f i x K_{2}$ 
A

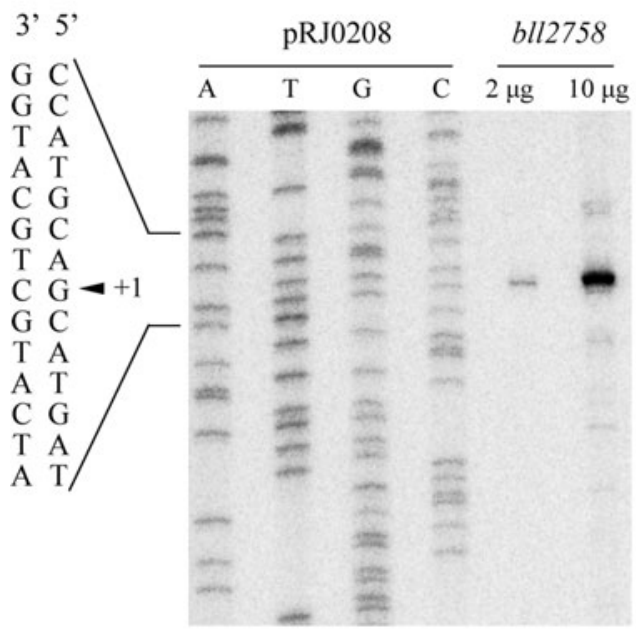

B

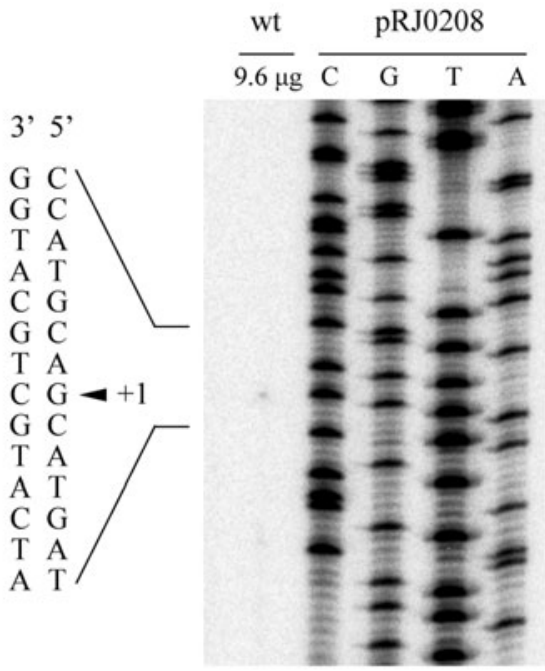

C

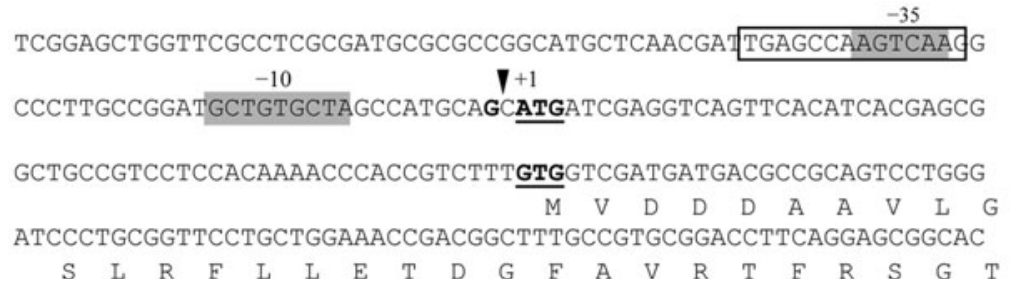

Fig. 4 Transcription start-site determination of bll2758 by primer extension, and analysis of the promoter region. a RNA synthesized by in vitro transcription was used. b Total RNA from micro-aerobically grown B. japonicum wild-type cells was used. The amounts of RNA applied are indicated above the lanes. Extension products were obtained with the $\left[{ }^{32} \mathrm{P}\right]$-labeled primers 9519-1 (a) and LR21 (b), and they were separated on a $6 \%$ denaturing polyacrylamide gel. The sequencing ladders were generated with plasmid pRJ0208 and primers 9519-1 (a) and LR21 (b). Part of the promoter region is

gene expression as compared with the wild type, whereas there was strong fix $K_{2}$ de-repression in the fix $K_{2}$ mutant, testifying the negative autoregulation phenomenon. In conclusion, the NnrR protein also does not appear to be involved in the negative control of fix $K_{2}$.

\section{Discussion}

Most of the work reported here deals with the B. japonicum bll2758 gene which codes for a FixT-like single-domain response regulator (Jenal and Galperin 2009). Proteins of that class interfere with other regulators through proteinprotein contact instead of binding to critical sites on DNA. A B. japonicum bll2758 null mutant, in contrast to fixJ and fix $K_{2}$ mutants, does not have a conspicuous phenotype with regard to symbiosis and nitrogen fixation (Anthamatten and Hennecke 1991; Nellen-Anthamatten et al. 1998). A priori, this does not preclude a negative regulatory role of the shown in the left margin of each panel, with the transcription start site $(+1)$ indicated by an arrowhead. c DNA sequence of the bll2758 promoter region. Again, the transcription start site $(+1)$ is indicated by an arrowhead. The newly proposed (GTG) and the annotated (ATG) translational start codons are underlined. The likely -10 and -35 promoter regions are shaded in gray, and the predicted FixK binding site is boxed. The beginning of the Bl12758 amino acid sequence is indicated in one-letter code

B112758 protein in the wild type because, even if knocking the protein out by mutation unleashes its targets, the effect would not necessarily be detectable in the form of a physiologically distinct phenotype. Much more compelling was the microarray experiment done with the bll2758 mutant, which clearly showed that none of the FixJ- or FixK $_{2}$-regulated targets are derepressed.

In conclusion, our work has revealed unexpected species-specific differences in the design of an oxygenresponsive signaling network despite the fact that the regulatory modules (FixL, FixJ, FixT, FixK) are conserved. Particularly puzzling in B. japonicum is the FixK $_{2}$ dependency of bll2758 gene expression although its product does not play the role FixT plays in S. meliloti or C. crescentus. The reason why bll2758 is activated by $\mathrm{FixK}_{2}$ remains enigmatic. Yet, negative autoregulation of fix $K_{2}$ gene expression does exist in B. japonicum and, therefore, asks for an alternative mechanistic explanation. While the available evidence suggests an indirect type of control, the 

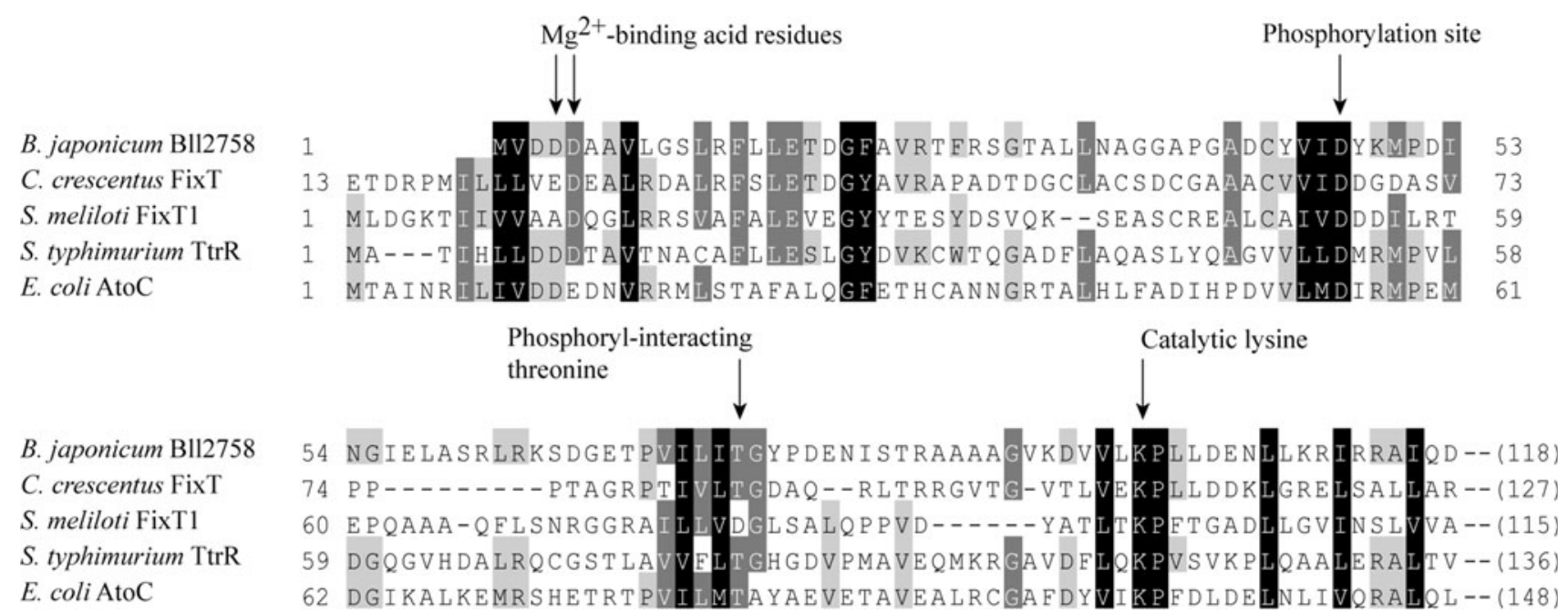

Fig. 5 Alignment of the B. japonicum B112758 amino acid sequence with other bacterial stand-alone receiver domains. Functionally important amino acid residues of receiver domains are indicated. The protein sequences and their GenBank identification numbers are: B. japonicum Bll2758, BAC48023; C. crescentus FixT, AAK22738;

Table 1 Microarray-assisted expression analysis of the 11 direct FixK $_{2}$ targets

\begin{tabular}{llll}
\hline $\begin{array}{l}\text { Gene } \\
\text { number }^{\mathrm{b}}\end{array}$ & $\begin{array}{l}\text { Gene } \\
\text { name }\end{array}$ & \multicolumn{2}{l}{$\begin{array}{l}\text { Fold-change of expression } \\
\text { compared to wild type }\end{array}$} \\
\cline { 3 - 4 } & & $\begin{array}{l}\text { Strain } 9043 \\
\left(\text { fix } K_{2}^{-}\right)\end{array}$ & $\begin{array}{l}\text { Strain 7414 } \\
(\text { bll2758 }\end{array}$ \\
\hline blr2763 & fixN & -101.9 & - \\
blr2767 & fixG & -63.3 & - \\
bll3998 & & -56.5 & - \\
blr4637 & hsp $C_{2}$ & -111.5 & - \\
blr4655 & $p p s A$ & -14.2 & -2.3 \\
bll6061 & fix $K_{1}$ & -19.1 & - \\
blr6062 & cycS & -45.9 & - \\
blr6070 & & -7.3 & - \\
bll6073 & phbC & -27.9 & - \\
bll7086 & hemN $N_{2}$ & -97.1 & - \\
bsr7087 & & -53.8 & - \\
\hline
\end{tabular}

a All strains were grown under micro-oxic conditions. Negative values indicate decrease of gene expression; - no change within the threshold fold-change range between +2 and -2

${ }^{\mathrm{b}}$ Gene numbers are from Rhizobase (http://genome.kazusa. or.jp/rhizobase). In the case of operons (e.g., fixNOQP) only the promoter-proximal gene is listed (i.e., blr2763, fixN)

possibility cannot be ruled out that Fix $\mathrm{K}_{2}$ represses its own gene directly. If this were the case, however, one would have to postulate a repressor binding motif somewhere around the fixK $K_{2} 5^{\prime}$ end that radically deviates from the otherwise highly conserved DNA binding site elaborated previously for FixK $\mathrm{K}_{2}$ at activated promoters (Mesa et al. 2005, 2008).
S. meliloti FixT 1 , AAK65326; S. typhimurium TtrR, CAB37412; E. coli AtoC, AAC75280. While the total number of amino acids of each protein is given at the far end in parentheses, a few amino acids from the carboxy-terminal ends are omitted

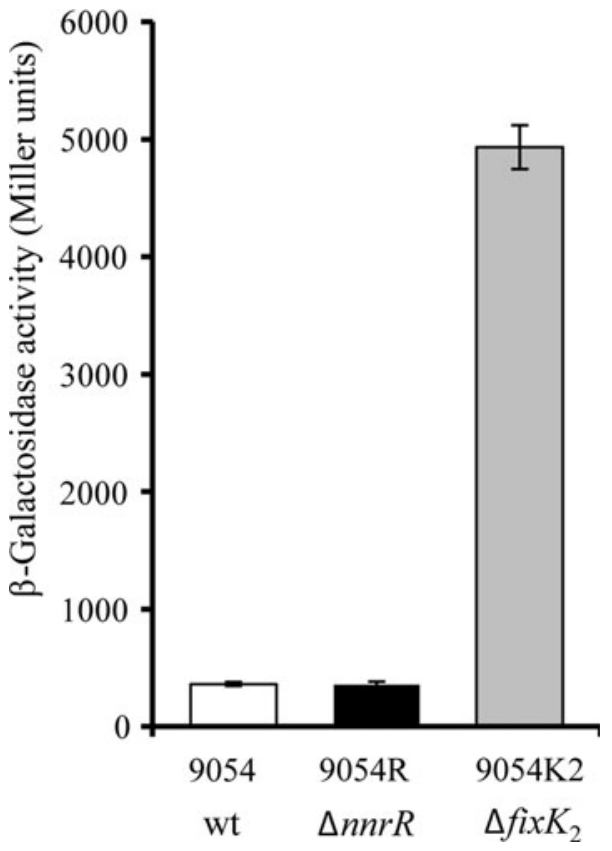

Fig. 6 Expression of a chromosomally integrated fix $K_{2}{ }^{\prime}-{ }^{\prime} l a c Z$ fusion in different $B$. japonicum backgrounds. The relevant genotype of host strains is indicated below strain designations; $w t$ wild type. Cells were grown micro-oxically for $72 \mathrm{~h}$ before $\beta$-galactosidase activity was determined. Values are the means \pm standard errors from at least three independent experiments with two cultures assayed in triplicate

Having excluded the Bll2758 protein as a negative effector, we will now have to focus on the other eight FixK $\mathrm{K}_{2}$-dependent genes that encode putative transcription factors. Three of them $\left(\mathrm{NnrR}, \mathrm{FixK}_{1}, \sigma^{54}\right)$ do not seem to be involved in down-regulating the fix $K_{2}$ gene. This leaves 
not less than five more regulatory genes (blr1216, bll2109, bll3466, bsr4636, blr7666) for analyses on their potential involvement in autoregulation. To assign such a function to one of them requires a considerable investment into future experimental work. According to the Kazusa annotation (http://genome.kazusa.or.jp/rhizobase) blr1216 codes for a Fur-like repressor, blr7666 for an AraC-like regulator, and bsr4636 for a kation transport regulator. The other two genes (bll2109, bll3466) code for members of the CRP/ FNR family of regulators. It will be of interest to find out if any of these regulators acts as a repressor of fix $K_{2}$ or, alternatively, as an activator of a gene that encodes a repressor of fix $K_{2}$.

Acknowledgments The crew of the Functional Genomics Center Zurich is acknowledged for help with the microarray analysis. Our research was supported by grants from the Swiss National Foundation for Scientific Research and the ETH Zurich.

\section{References}

Anthamatten D, Hennecke H (1991) The regulatory status of the fixLand fixJ-like genes in Bradyrhizobium japonicum may be different from that in Rhizobium meliloti. Mol Gen Genet 225:38-48

Anthamatten D, Scherb B, Hennecke H (1992) Characterization of a fixLJ-regulated Bradyrhizobium japonicum gene sharing similarity with the Escherichia coli fnr and Rhizobium meliloti fixK genes. J Bacteriol 174:2111-2120

Batut J, Boistard P (1994) Oxygen control in Rhizobium. Antonie van Leeuwenhoek 66:129-150

Beck C, Marty R, Kläusli S, Hennecke H, Göttfert M (1997) Dissection of the transcription machinery for housekeeping genes of Bradyrhizobium japonicum. J Bacteriol 179:364-369

Bergès $\mathrm{H}$, Checroun $\mathrm{C}$, Guiral S, Garnerone AM, Boistard P, Batut J (2001) A glutamine-amidotransferase-like protein modulates FixT anti-kinase activity in Sinorhizobium meliloti. BMC Microbiol 1:6

Crosson S, McGrath PT, Stephens C, McAdams HH, Shapiro L (2005) Conserved modular design of an oxygen sensory/signaling network with species-specific output. Proc Natl Acad Sci USA 102:8018-8023

Dixon R, Kahn D (2004) Genetic regulation of biological nitrogen fixation. Nature Rev Microbiol 2:621-631

Fischer HM (1994) Genetic regulation of nitrogen fixation by rhizobia. Microbiol Rev 58:352-386

Fischer HM, Babst M, Kaspar T, Acuna G, Arigoni F, Hennecke H (1993) One member of a groESL-like chaperonin multigene family in Bradyrhizobium japonicum is coregulated with symbiotic nitrogen fixation genes. EMBO J 12:2901-2912

Foussard M, Garnerone AM, Ni F, Soupène E, Boistard P, Batut J (1997) Negative autoregulation of the Rhizobium meliloti fixK gene is indirect and requires a newly identified regulator, FixT. Mol Microbiol 25:27-37
Garnerone AM, Cabanes D, Foussard M, Boistard P, Batut J (1999) Inhibition of the FixL sensor kinase by the FixT protein in Sinorhizobium meliloti. J Biol Chem 274:32500-32506

Hauser F, Pessi G, Friberg M, Weber C, Rusca N, Lindemann A, Fischer HM, Hennecke H (2007) Dissection of the Bradyrhizobium japonicum $\mathrm{NifA}+\sigma^{54}$ regulon and identification of a ferredoxin gene $(f d x N)$ for symbiotic nitrogen fixation. Mol Genet Genomics 278:255-271

Jenal U, Galperin MY (2009) Single-domain response regulators: molecular switches with emerging roles in cell organization and dynamics. Curr Opin Microbiol 12:152-160

Kaneko T, Nakamura Y, Sato S, Minamisawa K, Uchiumi T, Sasamoto S, Watanabe A, Idesawa K, Iriguchi M, Kawashima K, Kohara M, Matsumoto M, Shimpo S, Tsuruoka H, Wada T, Yamada M, Tabata S (2002) Complete genomic sequence of nitrogen-fixing symbiotic bacterium Bradyrhizobium japonicum USDA110. DNA Res 9:189-197

Kullik I, Fritsche S, Knobel H, Sanjuan J, Hennecke H, Fischer HM (1991) Bradyrhizobium japonicum has two differentially regulated, functional homologs of the sigma 54 gene $($ rpoN). J Bacteriol 173:1125-1138

Lois AF, Weinstein M, Ditta GS, Helinski DR (1993) Autophosphorylation and phosphatase activities of the oxygen-sensing protein FixL of Rhizobium meliloti are coordinately regulated by oxygen. J Biol Chem 268:4370-4375

Mesa S, Bedmar EJ, Chanfon A, Hennecke H, Fischer HM (2003) Bradyrhizobium japonicum NnrR, a denitrification regulator, expands the FixLJ-FixK 2 regulatory cascade. J Bacteriol 185:3978-3982

Mesa S, Ucurum Z, Hennecke H, Fischer HM (2005) Transcription activation in vitro by the Bradyrhizobium japonicum regulatory protein FixK 2 . J Bacteriol 187:3329-3338

Mesa S, Hauser F, Friberg M, Malaguti E, Fischer HM, Hennecke H (2008) Comprehensive assessment of the regulons controlled by the FixLJ-FixK ${ }_{2}$-FixK ${ }_{1}$ cascade in Bradyrhizobium japonicum. J Bacteriol 190:6568-6579

Mesa S, Reutimann L, Fischer HM, Hennecke H (2009) Posttranslational control of transcription factor Fix $_{2}$, a key regulator for the Bradyrhizobium japonicum-soybean symbiosis. Proc Natl Acad Sci USA 106:21860-21865

Miller JH (1972) Experiments in molecular genetics. Cold Spring Harbor Laboratory Press, Cold Spring Harbor

Nellen-Anthamatten D, Rossi P, Preisig O, Kullik I, Babst M, Fischer HM, Hennecke H (1998) Bradyrhizobium japonicum FixK $_{2}$, a crucial distributor in the FixLJ-dependent regulatory cascade for control of genes inducible by low oxygen levels. J Bacteriol 180:5251-5255

Paul R, Jaeger T, Abel S, Wiederkehr I, Folcher M, Biondi EG, Laub MT, Jenal U (2008) Allosteric regulation of histidine kinases by their cognate response regulator determines cell fate. Cell 133:452-461

Regensburger B, Hennecke H (1983) RNA polymerase from Rhizobium japonicum. Arch Microbiol 135:103-109

Soberón M, Morera C, Kondorosi A, Lopez O, Miranda J (2001) A purine-related metabolite negatively regulates fixNOQP expression in Sinorhizobium meliloti by modulation of fixK expression. Mol Plant-Microbe Interact 14:572-576

Sousa EHS, Tuckerman JR, Gonzalez G, Gilles-Gonzalez MA (2007) A memory of oxygen binding explains the dose response of the heme-based sensor FixL. Biochemistry 46:6249-6257 